\title{
Chemical Considerations Related to The Dilution of Commercial 10\% Povidone-Iodine For Use In The COVID-19 Pandemic
}

\author{
Colin Nuckolls
}

Sheldon and Dorothea Buckler Professor of Material Science

Department of Chemistry, Columbia University, New York, NY 10027

cn37@columbia.edu

http://nuckolls.chem.columbia.edu/

\begin{abstract}
While personal protective equipment is crucial in the fight against COVID-19, there are additional recommendations and protocols being instituted using nasal and oral antisepsis as an adjunct to help prevent viral spread. Povidone-iodine is a well-known antiviral agent commercially available in the US at concentrations between 5\%-10\%. Repeated use of lower-concentration povidoneiodine is frequently being implemented in infection control measures using nasal and oral antisepsis. This brief report describes the chemistry of dilute povidone-iodine solutions and highlights the challenges that exist in simply diluting the commonly available commercial $5-10 \%$ povidone-iodine solutions. Safety and efficacy could be improved with solutions specifically manufactured at lower concentration with processes that considered the multiple equilibria involved in povidone-iodine solutions, the $\mathrm{pH}$ of the effective solutions, and the effects of salts and other excipients in the formulation.
\end{abstract}

\section{Introduction}

Much of the world is mired in a pandemic caused by the spread of COVID-19. My laboratory and many others are intensely interested in developing processes and protocols to safely reopen in situations where adequate social distancing may not be possible. In a large, experimental chemistry and materials science research laboratory like mine, graduate students, post-docs, undergrads, staff scientists and administrative personnel work very closely together. Social distancing is a challenge when much of the work involves complex machinery, specialized analytical devices, limited space and collaborative bench work that is hard to physically separate. As we have searched for strategies in addition to social distancing that would enable safe re-opening of our labs, it is clear that personal protective equipment (PPE) has a primary role ${ }^{1}$. Chemists are very familiar with PPE as we are required to wear lab coats, safety glasses and gloves in synthetic labs and other types of PPE are routinely used when we deal with potentially noxious reaction processes. But what do we do when every person and every process could potentially expose our staff to transmission risk of COVID -19? As we have recently learned, asymptomatic lab staff could be actively infected with high viral loads in the nose and mouth ${ }^{2}$ with the potential to transmit infectious virus particles through aerosols, droplets and other dispersive elements ${ }^{3}$.

It is now clear that although PPE is important, it is not enough. As chemists, we naturally gravitate to chemical solutions, and in fact what we have found is that chemical antisepsis seems to have a critical role. Povidone-iodine (PVP-I), at concentrations low enough to be safely administered to the sites of high viral shedding in the mouth and nose, is emerging as a potentially useful nasal and oral antiseptic ${ }^{4}$. There is ample support for the rapid antiviral efficacy and for the safety of PVP-I 
at concentrations below about $2.0 \%{ }^{5}$. Most PVP-I solutions commercially available in the US are in the 5\%-10\% concentration range, though newer products such as Halodine Nasal Antiseptic and Halodine Oral Rinse (Halodine LLC, Spring House, PA) are specifically being manufactured and offered at low concentrations. Reports of the efficacy and safety of PVP-I at low concentrations have led many physicians and clinicians to advocate for the simple dilution of commercially available $10 \%$ PVP-I solutions with water or saline to produce PVP-I in the $0.25 \%-1.5 \%$ concentration range ${ }^{6}$. There is growing consensus that PVP-I in concentrations below $2.0 \%$ is a powerful adjunct to PPE, but the use of commercially available 10\% PVP-I is unsafe for routine daily intranasal and intraoral use ${ }^{7}$. The strategy of simple dilution poses some risks based on chemical differences and equilibrium changes that could occur when diluting $10 \%$ commercial PVP-I to $0.5 \%$ "homemade" PVP-I in a doctor's office, hospital or other clinical or pharmacy setting. Summarized below are some of the issues that should be considered when diluting commercial 10\% PVP-I to lower concentrations for use with PPE as adjunct viral protection strategies.

\section{Discussion}

The main question we sought to understand is, "What if I water-down conventional 10\% PVP-I to make 'dilute' PVP-I? Isn't that the same thing?" The short answer here is, "No, a 10\% PVP-I solution diluted down to $1 \%$ or $0.5 \%$ is not the same as a PVP-I solution manufactured at $1 \%$ or $0.5 \%$." 8 There are some complicated reasons related to the instability that $\mathrm{pH}$ and ion concentration introduce into aqueous equilibrium iodine chemistry. There are also some uncomplicated reasons that are related to the unspecified inactive ingredients and variation in available iodine allowed by the US Pharmacopeia (USP) analytical standards which govern commercial PVP-I drug products. According to the USP standards, which are recognized by the FDA, a "10\% PVP-I Solution" can mean a lot of different things with many different chemical compositions and many different properties. Most importantly, commercial 10\% PVP-I is almost never actually $10 \%$ PVP-I. The USP standard requires that a "Topical Povidone-Iodine Solution" is defined by the analytical range of the labeled iodine content. A $35 \%$ variation of measured iodine can still be labeled as " $10 \%$ Povidone-Iodine Solution." The USP definition of " $10 \%$ Povidone-Iodine Solution" allows the solution to deliver anywhere between $85 \%$ and $120 \%$ of the labeled iodine concentration. See below, From USP 29-NF 24, page 1780:

"Povidone-Iodine Topical Solution is a solution of povidone-iodine. It contains not less than 85.0 percent and not more than 120.0 percent of the labeled amount of iodine (I). It may contain a small amount of alcohol."

Note also that the USP allows a "...small amount of alcohol." This means that any topical PVP-I solution could contain alcohol, even if says "alcohol-free" on the label. If alcohol does appear on the label, as it does in many conventional PVP-I products, the USP allows the amount of alcohol to vary by up to $20 \%$. So just based on the allowed variance in the USP standards, when you try to make a specific concentration of PVP-I starting from conventional 10\% PVP-I, it is impossible to make the target concentration. Without the capacity to perform analytical chemistry on the labeled "10\% PVP-I" at the time of dilution, and without the ability to subsequently analyze the target dilution, it is impossible to make a specific low-concentration PVP-I. 
After the variance allowed in the iodine content, consider the allowed variation in solvent and solute content. It is not required that a " $10 \%$ Povidone-Iodine" solution have a specific solvent content or solute content. Think of it like 80 proof vodka, 80 proof whiskey, and 80 proof gin: all of these solutions are $40 \%$ ethanol but none of them are the same. All one can say about them is, "They all have the same ethanol concentration which is $40 \%$." This is similar to the situation with commercial 10\% PVP-I. It could be purely aqueous, partially aqueous, alcoholic, oxygenated, halogenated, hydrocarbon - the only restriction is on the USP limits for residual volatile-organic compounds (VOCs) from the manufacturing process. There are no solute restrictions. The only claim about conventional 10\% PVP-I topical solutions that can be made is, "They all have the same PVP-I concentration, which is labeled at $10 \%$, but actually could be anywhere from $8.5 \%$ to 12\%." Commercial PVP-I often contains salts, counterions, detergents, penetration enhancers, preservatives, alkalinizing agents, acidifying agents, surfactants, buffers, additional iodophors, macromolecules, soaps, disintegrants, lubricants, and other inactive excipients that may be required dependent on the intended application site or packaging requirements. The effectiveness, utility and toxicity of any excipient will be dependent concentration and could be worse at higher dilutions. For example, if a 10\% PVP-I solution with added surfactant is simply diluted, the concentration of the surfactant would be decreased. The aggregation and self-assembly of any given surfactant is determined by its critical micelle concentration, which is very sensitive to the concentration of the surfactant. Dilution could promote unintended surfactant assembly which could impair mucociliary clearance in the nose or cause unpredictable intolerance when used orally. Just from these qualitative explanations, it is easy to understand that a $10 \%$ PVP-I solution diluted down to a lower concentration is not the same as a PVP-I solution manufactured at a lower concentration. When we consider the complicated chemistry of iodine in these solutions, the arguments against dilution become even more compelling.

Commercial PVP-I is sold at high concentration - usually $10 \%$ - because low concentrations are very unstable when packaged in commercial polymer material container systems ${ }^{9}$. Because the conventional PVP-I product concentrations are so high, typically chemical buffers are added to prevent tissue irritation when applied to patients. Buffers are also added to maintain the $\mathrm{pH}$ of the commercial solutions in the range required by the USP analytical standards. The buffering capacity $(\beta)$ of a solution is governed by the concentration of the protons and the concentration of the conjugate base of the buffer $\left(C_{B}\right)$, which are governed by the $K a$ of the acid used in the buffer system:

$$
\beta=2.303\left[\frac{k_{w}}{\left[\mathrm{H}^{+}\right]}+\left[\mathrm{H}^{+}\right]+\frac{\left[\mathrm{H}^{+}\right] C_{B} K_{a}}{\left(\left[\mathrm{H}^{+}\right]+K_{a}\right)^{2}}\right]
$$

When commercial PVP-I at $10 \%$ is diluted with water or saline, it continues to have significant buffering capacity as the formula above clearly indicates. This causes the relative $\mathrm{pH}$ of the lowconcentration PVP-I to be maintained at the same elevated level as in the commercial undiluted PVP-I product. Normally, this would not be a problem for a conventional pharmaceutical product. But for PVP-I solutions, this is disastrous. PVP-I stability at low concentration requires an acidic environment. Alkaline or neutral conditions rapidly destabilize the iodine content in dilute solutions. It has been well described for decades in the solution chemistry of PVP-I that buffered solutions of aqueous PVP-I are particularly unstable at low concentrations. The use of buffers in 
low-concentration PVP-I solutions must be strictly avoided. The instability arises from the aqueous solution equilibrium that is established at low-concentrations for complexed and non-complexed iodine, iodide, and triiodide as shown below.

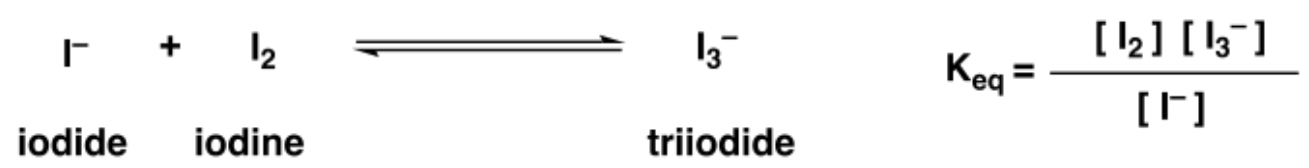

This condition, governed by the equilibrium constant $\left(\mathrm{K}_{\mathrm{eq}}\right)$, determines the relative and absolute concentrations of iodine, iodide, and triiodide. The equilibrium constant $\mathrm{K}_{\mathrm{eq}}$ is exquisitely sensitive to temperature, pressure, container system, exposure to light, counterion, buffering capacity, osmolarity and most importantly, $\mathrm{pH}$. To ensure stability, the solution components must be optimized to be in the most stable $\mathrm{pH}$ range for low concentrations of PVP-I which creates a favorable equilibrium. If commercial 10\% PVP-I is diluted, the solution will maintain significant buffering capacity and will be buffered well outside the stable $\mathrm{pH}$ range. Any freshly diluted solutions with residual buffering capacity will drive the unfavorable equilibrium reaction and rapidly result in solutions that are unstable, possibly toxic and rapidly ineffective. For dilution of commercial PVP-I to be safe and effective, upon dilution the residual buffering capacity would need to be immediately neutralized. This would be difficult achieve without knowing the identity and quantity of the precedent solution's acid buffer/conjugate base pair. The $\mathrm{pH}$ would need to be precisely and accurately measured, corrected with a standardized stock solution, ideally one specific for the buffer employed, and then remeasured periodically throughout the day to ensure durable correction. Periodic analysis should also be carried out on freshly diluted PVP-I to monitor effects of temperature, pressure, light exposure, ion drift and container effects.

The pure aqueous solution equilibrium of PVP-I is additionally complicated by dilution if we consider the other equilibria associated with the iodine species in aqueous media. The table below details the most important reactions. Because water and protons are present in these equilibria reactions, the amount of iodine, iodide, and triiodide will be critically dependent on all the factors described above and especially dependent on concentration and $\mathrm{pH}$. 
Table 1. lodine-containing species in aqueous iodine solutions: reactions and equilibria

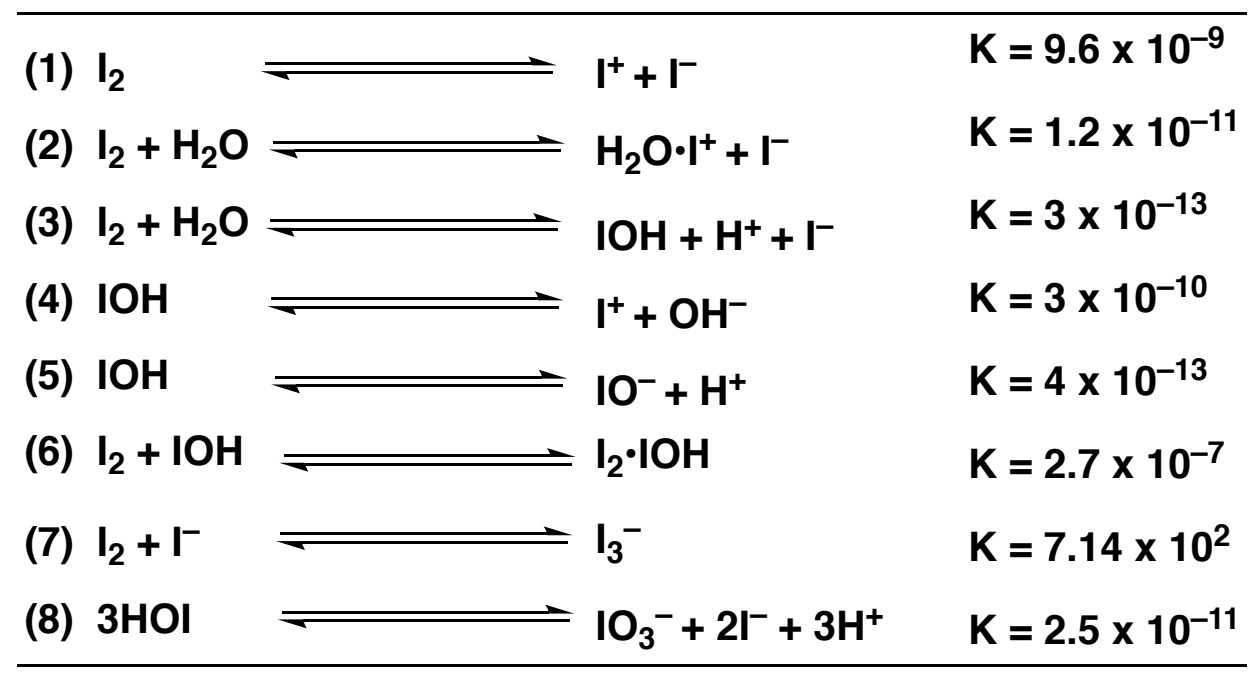

From Rackur, Journal of Hospital Infection 1985, 6, 13-23

The equilibria above are in idealized polymer-free, iodophor-free pure aqueous solutions of iodine. The simple iodine reactions above are further modified by polymer kinetics and polymer dynamics that we know are relevant when aqueous iodine is further complexed with the polyvinylpyrrolidone backbone (the "povidone" of "povidone-iodine") ${ }^{10}$. Considering the reversible reaction with the polymer host leads to the well-known paradoxical concentration-dependent biocidal effects. The figure below displays the chemical structure of the main species in solution when PVP-I is dissolved in water. This is a complex of $\mathrm{H}^{+} \mathrm{I}_{3}{ }^{-}$and adjacent residues on the PVP polymer.

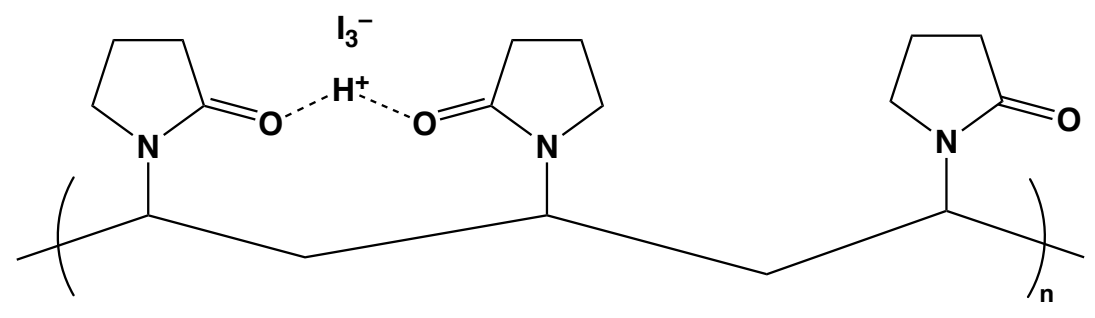

Even the chemical structure above is an oversimplification of the condition at any given concentration of PVP-I in aqueous solution. The equations below detail the two main complexes in PVP-I and their associated equilibria. Equation (1) is for the complex of $\mathrm{H}^{+} \mathrm{I}_{3}{ }^{-}$with PVP and Equation (2) describes $\mathrm{I}_{2}$ complexed with PVP. When the complexes dissociate in aqueous solution, the iodine species enter the various equilibria with water, protons, and other salts in solution. Simple dilution of commercial 10\% PVP-I solutions will alter these equilibria in unpredictable ways. Each individual commercial PVP-I "recipe" will cause unique equilibrium changes with idiosyncratic results but predictable instability. When conventional 10\% PVP-I is diluted, the buffers alone are enough to destabilize the target solutions, but all of the additives and excipients will also contribute to the disruption of the PVP-I solution equilibria. 
(1)

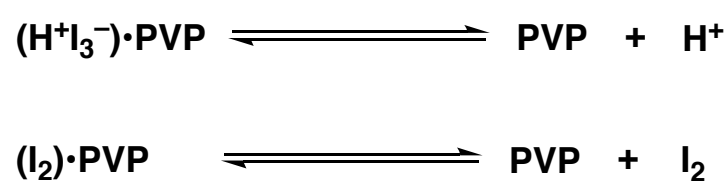

There are osmotic consequences that come with dilution which are important in nasal physiology. Dilution of conventional high-concentration PVP-I solutions, without knowing the precise concentrations of all excipients, will result in solutions that are not isosmotic with nasal fluid. A PVP-I solution with a demonstrated osmolarity of $\sim 300 \mathrm{mOsmol} / \mathrm{kg}$ of the dose intended for nasal application would be compatible with the osmotic conditions of the nasal secretions and the extracellular nasal mucosal space, and ideal for intranasal use. If conventional 10\% PVP-I is simply diluted with water or saline, it would be impossible to match the desired osmolarity without knowing the precise content and concentrations for all the potential additives delineated above. Even assuming there were minimal additives, matching the osmolarity would be almost impossible without some benchtop analytical chemistry available in the proposed setting of use. It would be difficult to adjust the osmolarity of the diluted PVP-I solution in a way that would allow rapid use in a clinical setting.

As described above, there is almost never a true "10\% PVP-I " solution which means you can never reliably produce a precise target concentration through dilution. Uncertainty in the concentration is probably not critical when employed as a one-time application on intact skin, for example, in preparation for surgery. The imprecision does cause complications when used repeatedly on more sensitive mucosal surfaces. The uncertainty in the labeled PVP-I concentrations is further magnified by the uncertainty in the dilutional volumes. Unless the dilution is performed with volumetric glassware or other calibrated quantifiable addition and mixing vessels, the cascade of uncertainties in concentration, content and volume will lead to less predictable results when finally applied to patients. The only way to ensure the safety of these dilutions would be to analyze them prior to use. Ideally, any target dilutions should be analyzed according to the USP method (USP 29-NF24, p1779). The preferred method for concentration of PVPI involves a thiosulfate reduction shown in the equation below.

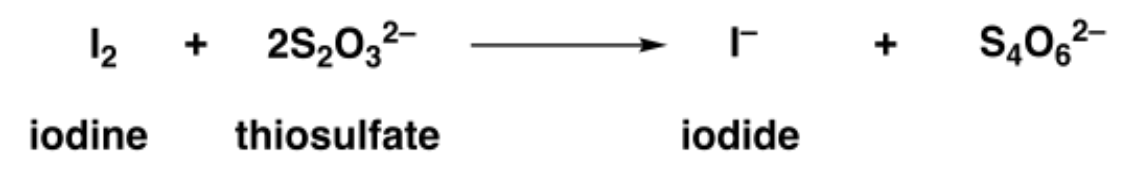

The iodine is a golden brown color and when the thiosulfate is added, it reduces the iodine to iodide. As the titration progresses, when the solution becomes a light yellow, a starch solution is added to create a starch-iodine complex that is blue/black in color. The titration continues until the solution turns colorless. The starch solution degrades quickly and would need to be made fresh prior to the analysis. Done correctly, the titration method requires flasks, burettes, and other precision laboratory glassware, materials impractical to maintain in a clinical setting. Notwithstanding the inconvenience and cost of establishing the processes and procedures required to accurately determine the safety and composition of freshly diluted PVP-I solutions, a routine dilution program would require significant staff training or the addition of an additional staff member trained in analytical chemistry. 


\section{Conclusion}

The simple on-site dilution of $10 \%$ PVP-I with water or saline solution to produce solutions in the $0.25 \%-2.0 \%$ range should be viewed with caution and undertaken only with the full appreciation of the risks detailed above. Fortunately in our laboratories, we would be able to employ diluted commercial solutions with the appropriate subsequent analysis, monitoring and storage conditions though this is inconvenient and impractical. PVP-I solutions manufactured and designed to be safe, stable and physiologically compatible in concentrations between $0.25 \%-2.5 \%$, are preferred for repeated intraoral and intranasal use as chemical adjuncts to PPE.

${ }^{1}$ Chu DK, Akl EA, Duda S, et al. Physical distancing, face masks, and eye protection to prevent personto-person transmission of SARS-CoV-2 and COVID-19: a systematic review and meta-analysis. Lancet. 2020; S0140-6736(20)31142-9.

${ }^{2}$ Gandhi M, Yokoe DS, Havlir DV. Asymptomatic transmission, the Achilles' heel of current strategies to control Covid-19. N Engl J Med. 2020. Published online on April 24, 2020.

${ }^{3}$ Mittal R, Ni R, Jung-Hee Seo. The flow physics of COVID-19. 2020; J. Fluid Mech:894 (F2).

${ }^{4}$ Parhar HS, Tasche, K, Brody, RM, et al. Topical preparations to reduce SARS-CoV-2 aerosolization in head and neck mucosal surgery. Head \& Neck 2020; 42:1268- 1272.

${ }^{5}$ Bidra AS, Pelletier JS, Westover JB, Frank S, Brown SM, Tessema B. Rapid In-Vitro Inactivation of Severe Acute Respiratory Syndrome Coronavirus 2 (SARS-CoV-2) Using Povidone-Iodine Oral Antiseptic Rinse. Journal of Prosthodontics 2020. doi:10.1111/jopr.13209.

${ }^{6}$ Challacombe S, Kirk-Bayley J, Sunkaraneni V, et al. Povidone iodine. Br Dent J. 2020; 22: 656-657.

${ }^{7}$ Frank S, Capriotti J, Brown SM, Tessema, B. Povidone-Iodine Use in Sinonasal and Oral Cavities: A Review of Safety in the COVID-19 Era. Ear, Nose \& Throat Journal 2020.

https://doi.org/10.1177/0145561320932318.

${ }^{8}$ Zamora JL. Chemical and microbiologic characteristics and toxicity of povidone-iodine solutions.

Am J Surg 1986; 3:400-6. doi: 10.1016/0002-9610(86)90477-0.

${ }^{9}$ Bhagwat et al. Stabilizing Packaged Iodophor And Minimizing Leaching Of Iodine

Through Packaging, US Patent 4,996,048.

${ }^{10}$ Rackur H. New aspects of mechanism of action of povidone-iodine. J Hosp Infect 1985; 6 Suppl A:1323. doi:10.1016/s0195-6701(85)80041-4. 